\title{
Chlormethiazole or chlordiazepoxide in alcohol detoxification
}

\author{
Denise Duncan and David Taylor
}

The alcohol withdrawal syndrome is characterised by symptoms such as anxiety, insomnia. anorexia, nausea, tremor, sweating and disturbances in coordination. These symptoms tend to occur within 6 to 12 hours of alcohol-dependent patients stopping or reducing their alcohol intake. Less frequently, convulsions, hallucinosis and delirium tremens can occur. Delirium tremens (disturbed orientation, visual and auditory hallucinations, paranoid ideation and tremulousness) is the most serious complication of alcohol withdrawal and occurs in up to $5 \%$ of patients withdrawing from alcohol. If it is severe or poorly treated, death can occur. In the UK some clinicians use chlormethiazole while others favour chlordiazepoxide. This article will examine and compare the use of these two drugs.

In the early 1960s, chlormethiazole was introduced for the management of acute alcohol withdrawal. Derived from the thiazole moiety of thiamine, it has sedative, hypnotic and anticonvulsant properties (although it seems to have little effect in the prevention of seizures (Dollery, 1991)). Chlormethiazole appears to work by enhancing gamma-aminobutyric acid (GABA) transmission. It is rapidly absorbed after oral administration but undergoes extensive presystemic metabolism in the liver. The systemic bioavailability is therefore unpredictable but greatly increased in patients with cirrhosis. Chlormethiazole is a short acting drug with a plasma half-life of approximately 4 hours; although this may be increased to up to 9 hours in advanced liver disease and up to 12 hours in the elderly.

\section{Efficiency trials}

Until the introduction of chlormethiazole, alcohol detoxification involved the use of drugs such as neuroleptics, barbiturates, chloral hydrate or paraldehyde. In the 1960s and 1970s, trials comparing chlormethiazole with neuroleptics, barbiturates and benzodiazepines found chlormethiazole to be at least as effective as these drugs (Majumdar, 1990) and subsequently chlor- methiazole gained favour in Europe. Many of these trials were open studies. In the 1980s, double-blind comparator trials also found chlormethiazole to be more effective than bromocriptine (Burroughs et al, 1985) and clonidine (Robinson et al, 1989). Meanwhile, in America, Kaim et al (1969), showed that chlordiazepoxdde was more effective than both neuroleptics and thiamine; this resulted in chlordiazepoxide's widespread use there.

Since the 1970s there have been three trials comparing chlordiazepoxide and chlormethiazole. In the first, a double-blind study by McGrath (1975), 100 consecutive admissions were randomised to either chlordiazepoxide or chlormethiazole. In the first 24 hours $100 \mathrm{mg}$ of chlordiazepoxide or four capsules of chlormethiazole were given 6 hourly. The dose was subsequently reduced by $25 \mathrm{mg}$ of chlordiazepoxide or one capsule of chlormethiazole per dose per day depending on the patient's condition. In the first 7 days, 14 patients stopped treatment with chlordiazepoxide compared to seven patients stopping chlormethiazole treatment. Discontinuing treatment was blamed on feelings of tension or restlessness or the development of delirium tremens. Forty-one patients presented with or developed withdrawal symptoms with chlordiazepoxide compared with 46 patients on chlormethiazole. Although four patients developed delirium tremens with chlordiazepoxide compared with no patients on chlormethiazole, this was not statistically significant. Both drugs were considered to be equally effective.

One drawback of this trial was that the author's definition of delirium tremens included so-called 'pre-delirius states'. Also, once the code was broken and subjects were found to be on chlordiazepoxide, they were automatically swapped to chlormethiazole. If found to be on chlormethiazole they were more likely to be kept on their trial regime. This perhaps indicates some bias and possibly affected the trial's results.

In a second randomised double-blind trial by Lapierre et al (1983), 40 patients with moderate to severe symptoms received either chlormethiazole 
or chlordiazepoxide for 7 days. Doses were adjusted to treat the patient's symptoms but no more than $400 \mathrm{mg}$ of chlordiazepoxdde or 16 capsules of chlormethiazole were allowed over a 24 hour period. Both drugs were shown to be effective and there was no statistically significant difference between the two drugs.

In the third double-blind trial (Burroughs et al, 1985), 71 patients were allocated to either a minor withdrawal study group (44 patients) or a major withdrawal study group (27 patients). Patients were randomly assigned to either bromocriptine, chlordiazepoxide, chlormethiazole or placebo in the minor withdrawal group and to bromocriptine, chlordiazepoxide or chlormethiazole in the major withdrawal group. Chlormethiazole and chlordiazepoxide were found to be significantly more effective than bromocriptine. No difference was found between chlordiazepoxide and chlormethiazole.

It is perhaps noteworthy that there were no power calculations for any of these trials, and so it is possible that subject numbers were too small to show a difference between chlordiazepoxide and chlormethiazole even though one existed.

\section{Adverse effects}

Adverse effects of chlormethiazole are generally mild and include transient nasal congestion and irritation. Increased nasopharyngeal or bronchial secretions have also occurred and this could be dangerous in a heavily sedated patient (Majumdar, 1990). Because of chlormethiazole's sedative properties, a reducing daily dose is given to avoid accumulation and over-sedation. Accumulation is especially likely to occur in patients with liver disease as the bioavailability of the drug is increased since metabolism is reduced. These patients are also more susceptible to the drug's sedative effects because of increased cerebral sensitivity. Patients with moderate liver disease should therefore be prescribed lower initial doses.

Chlormethiazole used long-term at high doses has produced problems of physical dependence and withdrawal symptoms (Hession et al, 1979). Perhaps as a consequence of this, it is recommended that chlormethiazole is not given for longer than 9 days when treating alcohol withdrawal symptoms (Association of the British Pharmaceutical Industry, 1995). More serious adverse effects have also been reported and these include "coma, respiratory depression, hypotension, hypothermia and death" (Erstad \& Cotugno, 1995). These problems are especially likely to occur if patients continue to misuse alcohol (McInnes, 1987). Because of this, the manufacturer. Astra, states that patients should be treated in hospital. Only in exceptional circumstances should they be treated as out-patients by specialised units and even then only if the daily dose is monitored closely by community health staff (ABPI, 1995). Chlormethiazole should never be given to patients who continue to drink.

Chlordiazepoxide, a long-acting benzodiazepine, has anxiolytic, sedative and hypnotic properties as well as being a muscle relaxant and an anticonvulsant. It has $a$ half-life of approximately 15 hours (range 5-30) and it is metabolised to active metabolites which have half-lives of up to 60 hours (Dollery, 1991). As with chlormethiazole, a reducing dose should be given to avoid accumulation and over-sedation and lower doses should be given in liver disease. Dependence and withdrawal symptoms can occur with chlordiazepoxide but generally only after long-term use. In trials, chlordiazepoxide was generally well tolerated and it is considered to be fairly safe in overdose. Importantly, if respiratory depression does occur it can be reversed with flumazenil.

\section{Summary}

To date chlordiazepoxide and chlormethiazole have both been used widely to treat alcohol withdrawal symptoms. They appear to be equally effective but there are few comparative data available and some of what is available is flawed. Chlordiazepoxide does seem to be the safer alternative since chlormethiazole has a much narrower safety margin and is considered dangerous in patients who are still misusing alcohol. Because of this, the CRAG/SCOTMEG Working Group on Mental Illness in their good practice statement (1994) do not support the use of chlormethiazole. As mentioned previously, chlormethiazole has highly variable bioavailability which makes the effect of a given dose difficult to predict in an individual, and it is not licensed for use in out-patients unless the daily dose is being monitored closely. Because of the restrictions on chlormethiazole's use and the problems associated with it, chlordiazepoxide should therefore be the preferred drug in alcohol detoxification.

\section{References}

Assoclation OF THE BRTISH PHARMaceUtical INDUSTRY (1995) ABPI Data Sheet Compendium. London: Datapharm Publications Limited.

Burroughs, A. K., MORGan, M. Y. \& SHERLOCK, S. (1985) Double-blind controlled trial of bromicriptine. chlordiazepoxide and chlormethiazole for alcohol withdrawal symptoms. Alcohol and Alcoholism; 20. 263-271.

CRAG/SCOTMEG WORKING GROUP ON MENTAL ILLNESS (1994) The Management of Alcohol Withdrawal and Delirium Tremens - a Good Practice Statement. London: HMSO.

DoLlerY, C. (ed.) (1991) Therapeutic Drugs. New York: Churchill Ltvingstone. 
ERstad, B. L. \& Cotugno, C. L. (1995) Management of alcohol withdrawal. American Joumal of Health-System Pharmacy, 82, 697-709.

Hession M. A., Verma, S. \& MOHAN BhaKta, K. G. (1979) Dependence on chlormethlazole and effects of its withdrawal. Lancet, $t$, 953-954.

KAIM, S. C., KLETT, C. J. \& ROTHFELD, B. (1969) Treatment of the acute alcohol withdrawal state: a comparison of four drugs. American Joumal of Psychiatry, 125, 1640-1646.

LAPIERRE, Y. D., BULMER, D. R., OYEWUM, M. L., et al (1983) Comparison of chlormethiazole (Heminevrin) and chlordiazepoxdde (Lbrium) in the treatment of acute alcohol withdrawal. Neuropsychobiology, 10, 127-130.

MAJUMDAR, S. K. (1990) Chlormethiazole: current status in the treatment of the acute ethanol withdrawal syndrome. Drug and Drug Dependence, 27, 201-207.

MCGRATH, S. D. (1975) A controlled trial of chlormethiazole and chlordiazepoxdde in the treatment of the acute withdrawal phase of alcoholism. British Joumal of Addiction, 70, 81-90.

MCINNES, G. T. (1987) Chlormethiazole and alcohol: a lethal cocktail. British Medical Journal, 284, 592.

Robinson, B. J., Robinson, G. M. Manng, T. J. B. et al (1989) Is clonidine useful in the treatment of alcohol withdrawal? Alcoholism: Clinical and Experimental Research, 18, 95-98.

Denise Duncan, Senior Drug Information Pharmacist: *David Taylor, Chief Pharmacist. The Maudsley Hospital, Denmark Hill, London SE5 8AZ

*Correspondence

\title{
Treatment options for rapid-cycling bipolar affective disorder
}

\author{
David Taylor and Denise Duncan
}

Rapid cycling (RC) occurs in up to $20 \%$ of patients with bipolar affective disorder and is particularly common in women. It is generally defined as a condition where four or more episodes of (hypo-) mania or depression occur in any year. Frequently, patients with $\mathrm{RC}$ switch rapidly between episodes without passing through a euthymic stage. RC is notoriously difficult to treat and the refractoriness of the condition has led to a number of drugs and combinations being put forward as potential therapeutic interventions. This article reviews current treatments and suggests a treatment strategy based on avallable evidence of efficacy and tolerability.

\section{Lithium}

Lithium is the mainstay of the treatment of bipolar affective disorder as a whole, where it is effective in around two-thirds of patients (Prien \& Gelenberg, 1989). Many of those that do not respond to lithium are rapid-cyclers; indeed lithium's relatively poor efficacy in RC has long been known (Dunner \& Fleve, 1974) and average treatment failure rates in RC may be as high as around 80\% (Calabrese \& Woyshville, 1995). Nevertheless, lithium's safety and tolerability are well-established and so it remains a drug of first choice, even in RC. Its observed poor efficacy in some patients, however, has forced the development of other therapies.

\section{Carbamazepine}

Carbamazepine is now widely used in a variety of mood disorders and is probably at least as effective as lithium in most aspects of bipolar affective disorder (e.g. Shaw, 1988; Small et al, 1991, among many others). The efficacy of carbamazepine in RC is well-established. Joyce (1988), in an open trial of six months' duration. found that a small proportion (2/12) of lithium non-responders with RC showed a useful response to carbamazepine alone and several more $(5 / 12)$ responded to carbamazepine plus lithium. Calabrese \& Woyshville (1995) abstracted data on 